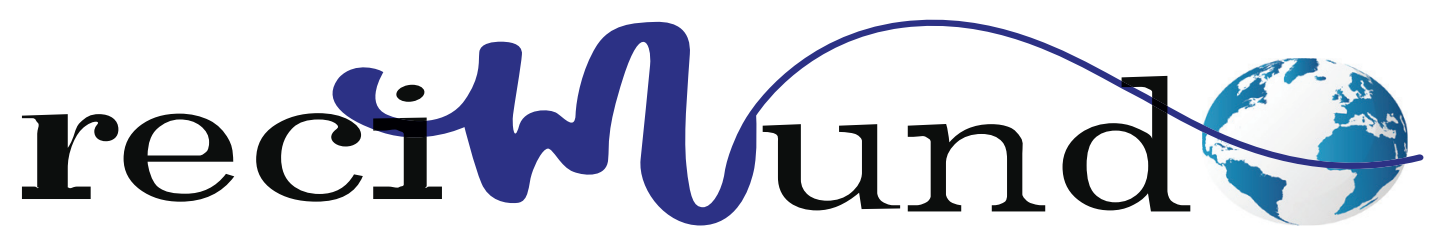

Revista Científica Mundo de la Investigación y el Conocimiento

DOI: $10.26820 /$ recimundo/5.(1).enero.2021.90-98

URL: http://recimundo.com/index.php/es/article/view/987

EDITORIAL: Saberes del Conocimiento

REVISTA: RECIMUNDO

ISSN: 2588-073X

TIPO DE INVESTIGACIÓN: Artículo de Revisión

CÓDIGO UNESCO: 3205 Medicina Interna

PAGINAS: 90-98

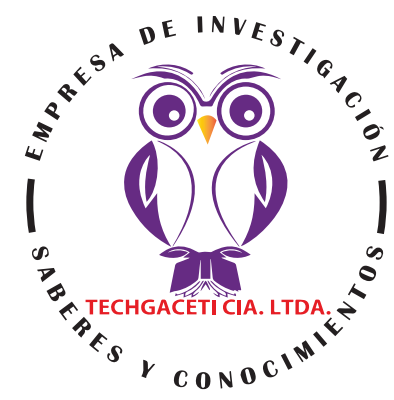

\title{
Búsqueda activa de personas vulnerables para garantizar una atención integral en tiempos de pandemia
}

Active search for vulnerable individuals to ensure comprehensive care

in times of pandemic

Busca ativa de pessoas vulneráveis para garantir cuidados abrangentes em tempos de pandemia

Pedro Javier Baquerizo Suárez; David Adrián Zamora Baquerizo²; Leydi Diana Moreira Cedeño³

RECIBIDO: 28/11/2020 ACEPTADO: 06/12/2020 PUBLICADO: 31/01/2021

1. Magister en Gerencia de Salud para el Desarrollo Local; Médico; Administrador Educativo; Investigador Independiente, Guayaquil, Ecuador; pjbaquerizo@hotmail.com; (iD https://orcid.org/0000-0002-2700-7654

2. Médico; Investigador Independiente, Guayaquil, Ecuador; davidjudezb14@gmail.com; (iD https://orcid.org/0000-00033915-3090

3. Médico; Investigador Independiente, Guayaquil, Ecuador; dayanalinda_23@hotmal.com; (iD https://orcid.org/0000-00025704-9927

CORRESPONDENCIA

Danilo Francisco Esquivel Ramírez

danier15@outlook.com

Quito, Ecuador

() RECIMUNDO; Editorial Saberes del Conocimiento, 2021 


\section{RESUMEN}

En la actualidad, se ha hecho evidente que los distintos sistemas de salud del mundo entero, enfrenten pesadas cargas como resultado de la mortal enfermedad del coronavirus. En respuesta a esto, los países han venido planeando, ejecutando e imponiendo restricciones dirigidas a minimizar la transmisión del virus, buscándose con estas medidas prevenir que la pandemia genere consecuencias nefastas en la humanidad, especialmente los grupos que son los denominados vulnerables, a los que los ordenamientos jurídicos estatales e internacionales le aplican un sistema protector de derechos más intenso. Entiéndase por vulnerabilidad, aquella que se encuentra dada por razones inherentes a su condición biológica, física y mental. En este sentido, es necesario que las autoridades sanitarias brinden perspectivas Inter secciónales y atención a las necesidades y al impacto diferenciado de dichas medidas en los derechos humanos de los grupos históricamente excluidos o en especial riesgo, tales como: personas adultas mayores y personas de cualquier edad que tienen afecciones médicas preexistentes, personas privadas de libertad, mujeres, pueblos indígenas, personas en situación de movilidad humana, niñas, niños y adolescentes, personas afrodescendientes, personas con discapacidad, personas trabajadoras, y personas que viven en pobreza y pobreza extrema, especialmente personas trabajadoras informales y personas en situación de calle; así como en las defensoras y defensores de derechos humanos, líderes sociales, profesionales de la salud y periodistas. En virtud de esto, es fundamental que las naciones del mundo entero logren implementar políticas que encierren como punto único la prevención y justicia como principio bioético, especialmente aquellas medidas que logren prevenir a las poblaciones vulnerables, por ser éstas las más desprotegidas ante males de salud como éste, buscándose de esta forma garantizar en mayor grado un acceso universal e igualitario a los recursos sanitarios disponibles, evitando dispendio, entiéndase por dispendio, la desproporción en el uso del recurso.

Palabras clave: Personas Vulnerables; Pandemia; COVID 19; Medidas Sanitarias; Derechos Humanos.

\section{ABSTRACT}

Today, it has become apparent that the various health systems around the world face heavy burdens as a result of the deadly coronavirus disease. In response to this, the countries have been planning, executing and imposing restrictions aimed at minimizing the transmission of the virus, seeking with these measures to prevent the pandemic from generating dire consequences on humanity, especially the groups that are the so-called vulnerable, to whom State and international legal systems apply a more intense protection system of rights. Understand by vulnerability, one that is given for reasons inherent to their biological, physical and mental condition. In this sense, it is necessary for health authorities to provide intersectional perspectives and attention to the needs and differentiated impact of these measures on the human rights of historically excluded groups or at special risk, such as: older people and people of any age who have pre-existing medical conditions, people deprived of liberty, women, indigenous peoples, people in a situation of human mobility, girls, boys and adolescents, people of African descent, people with disabilities, working people, and people living in poverty and extreme poverty, especially people informal workers and people living on the streets; as well as human rights defenders, social leaders, health professionals and journalists. In virtue of this, it is of great importance that the nations of the entire world manage to implement policies that include prevention and justice as a single point as a bioethical principle, especially those measures that manage to prevent vulnerable populations, since they are the most unprotected in the face of health ills like this, seeking in this way to guarantee a greater degree of universal and equal access to available health resources, avoiding waste, understood by waste, the disproportion in the use of the resource.

Keywords: Vulnerable Persons; Pandemic; COVID 19; Health Measures; Human Rights.

\section{RESUMO}

Hoje, tornou-se evidente que os vários sistemas de saúde em todo o mundo enfrentam pesados encargos como resultado da doença mortal do coronavírus. Em resposta a isto, os países têm planejado, executado e imposto restrições visando minimizar a transmissão do vírus, buscando com estas medidas evitar que a pandemia gere conseqüências terríveis para a humanidade, especialmente para os grupos que são chamados vulneráveis, aos quais os sistemas jurídicos estatais e internacionais aplicam um sistema de proteção de direitos mais intenso. Compreender pela vulnerabilidade, que é dada por razões inerentes à sua condição biológica, física e mental. Neste sentido, é necessário que as autoridades sanitárias ofereçam perspectivas intersetoriais e atenção às necessidades e impactos diferenciados destas medidas sobre os direitos humanos de grupos historicamente excluídos ou em risco especial, como por exemplo: pessoas idosas e pessoas de qualquer idade que tenham condições médicas preexistentes, pessoas privadas de liberdade, mulheres, povos indígenas, pessoas em situação de mobilidade humana, meninas, meninos e adolescentes, pessoas de ascendência africana, pessoas com deficiência, trabalhadores e pessoas que vivem na pobreza e pobreza extrema, especialmente pessoas que trabalham informalmente e pessoas que vivem nas ruas; assim como defensores dos direitos humanos, líderes sociais, profissionais de saúde e jornalistas. Em virtude disto, é de grande importância que as nações do mundo inteiro consigam implementar políticas que incluam a prevenção e a justiça como um único ponto como princípio bioético, especialmente aquelas medidas que conseguem prevenir populações vulneráveis, já que são as mais desprotegidas diante de males de saúde como este, buscando desta forma garantir um maior grau de acesso universal e igualitário aos recursos de saúde disponíveis, evitando o desperdício, entendido pelo desperdício, a desproporção no uso do recurso.

Palavras-chave: Pessoas Vulneráveis; Pandemia; COVID 19; Medidas de Saúde; Direitos Humanos. 


\section{Introducción}

En la actualidad las autoridades sanitarias del mundo entero han creado programas e intervenciones comunitarias basadas en conceptos y bases de salud pública, donde se le da herramientas, conocimientos e igualdad de oportunidades para que en conjunto, bajo lineamientos comunitarios generales, "se les permita a las familias mejorar sus condicionantes de la salud y desarrollar las medidas sanitarias necesarias para evitar la propagación del Virus Mortal Coronavirus". (Jacobo, 2020)

A razón de lo mencionado, es necesario argumentar que a raíz de la llegada del COVID-19, se han ido brindando a las distintas poblaciones del mundo entero, "las herramientas necesarias para la prevención de este mal de salud, en especial a grupos altamente vulnerables, con el fin de que éstos logren su protección y autocuidado". (Jacobo, 2020). No obstante, en estos tiempos, en donde existen países con altos niveles de insalubridad, los cuales se caracterizan "por comprender un sistema de salud colapsado, débil, donde los que menos tienen son los que más sufren, muriendo muchas veces, por enfermedades totalmente prevenibles, es que se considera de gran valor fortalecer y promover una cultura preventiva". (Jacobo, 2020)

Se logran entender entonces que, "la pandemia del COVID-19 puede afectar gravemente la plena vigencia de los derechos humanos de la población en virtud de los serios riesgos para la vida, salud e integridad personal que supone esta enfermedad". (Levín, 2020). Así, como sus impactos de inmediato, mediano y largo plazo sobre las sociedades en general, y sobre las personas y grupos en situación de especial vulnerabilidad. De allí, que a través de la ejecución de medidas y planes de intervención de la salud a comunidades "se busque brindar a las distintas poblaciones las herramientas, conocimientos e igualdad de oportunidades para que en conjunto, bajo nuestra guía, mejoren los determinantes y/o condicionantes de su salud". (Levín, 2020). De más está decir que:

La implementación de estas medidas sanitarias a nivel mundial y con mayor vehemencia en países latinoamericanos busquen preservar la salud, concebida como bienestar, de grupos altamente vulnerables (enfermos crónicos, edad avanzada, nivel social bajo, bajo ingreso económico, locaciones marginadas, bajo nivel educativo, entre otros) a través del fortalecimiento de su empoderamiento comunitario. (Levín, 2020)

De igual forma, según la (CIDH, 2020), "estos programas y medidas sanitarias tienen como propósito: educar sobre las medidas de prevención, signos de alarma y autocuidado frente a la pandemia COVID-19". Y del mismo modo, pretendan reducir el riesgo de contagio, "a través de la distribución y redistribución de kits de higiene y protección a grupos altamente vulnerables y conservar la sostenibilidad económica familiar a través de la autogeneración de ingresos con enfoque de género, en grupos vulnerables". (CIDH, 2020)

En continuidad del tema, la ( $\mathrm{CIDH}, 2020)$, refiere que es fundamental resaltar que:

Las naciones latinoamericanas se encuentran caracterizadas por enfrentar crisis sanitarias, las cuales en su mayoría son originadas por las profundas brechas sociales en que la pobreza y la pobreza extrema "constituyen un problema transversal que se encuentra inherente en todos sus estados; siendo los mismos causados por la falta o precariedad en el acceso al agua potable y al saneamiento; la inseguridad alimentaria, las situaciones de contaminación ambiental y la falta de viviendas o de 
hábitat adecuado; motivos éstos a los que se suman altas tasas de informalidad laboral y de trabajo e ingresos precarios que afectan a un gran número de personas en la región y que hacen aún más preocupante el impacto socioeconómico del COVID-19; aspectos que analizados en su totalidad impiden o dificultan a millones de personas tomar medidas básicas de prevención contra la enfermedad, en particular cuando afecta a grupos en situación de especial vulnerabilidad. (p.8).

Por todo esto, se puede decir que la pandemia supone desafíos aún mayores para los países del mundo entero, en especial aquellos con mayores desafíos sanitarios y necesidades en políticas de salud acertadas, tanto en términos de medidas sanitarias, como en capacidades económicas, que permitan poner en marcha estrategias o lineamientos de atención y contención que resultan urgentes y necesarias para proteger efectivamente a sus poblaciones, acordes con el Derecho Internacional de los Derechos Humanos.

En virtud de estos señalamientos, se hace necesario destacar que las políticas sanitarias implementadas a nivel mundial, establecidas como medidas de contención, tienen el fin de enfrentar y prevenir los efectos de la pandemia, llevando, según sea el caso de cada Nación o necesidad en salud, a que las autoridades establezcan la suspensión y restricción de algunos derechos, los cuales, en algunos casos, se han declarado "estados de emergencia", "estados de excepción", "estados de catástrofe por calamidad pública", o "emergencia sanitaria", a través "de decretos presidenciales y normativa de diversa naturaleza jurídica con el fin de proteger la salud pública y evitar el incremento de contagios". (CIDH, 2020)
Asimismo, se han establecido medidas de distinta naturaleza que restringen los derechos de la libertad de expresión, el derecho de acceso a la información pública, la libertad personal, la inviolabilidad del domicilio, el derecho a la propiedad privada; "y se ha recurrido al uso de tecnología de vigilancia para rastrear la propagación del coronavirus, y al almacenamiento de datos de forma masiva". (CIDH, 2020).

\section{Metodología}

\section{Fuentes documentales}

El análisis correspondiente a las características que predomina en el tema seleccionado, llevan a incluir diferentes fuentes documentales encargadas de darle el respectivo apoyo y en ese sentido cumplir con la valoración de los hechos a fin de generar nuevos criterios que sirven de referencia a otros procesos investigativos. Para (Arias, 2010), las fuentes documentales incorporadas en la investigación documental o bibliográfica, "representa la suma de materiales sistemáticos que son revisados en forma rigurosa y profunda para llegar a un análisis del fenómeno". (p.41). Por lo tanto, se procedió a cumplir con la realización de una lectura previa determinada por encontrar aquellos aspectos estrechamente vinculados con la "Búsqueda activa de personas vulnerables para garantizar una atención integral en tiempos de pandemia" para luego explicar mediante un desarrollo las respectivas apreciaciones generales de importancia.

\section{Técnicas para la Recolección de la Infor- mación}

La conducción de la investigación para ser realizada en función a las particularidades que determinan a los estudios documentales, tiene como fin el desarrollo de un conjunto de acciones encargadas de llevar a la selección de técnicas estrechamente vinculadas con las características del estudio. En tal sentido, (Arias 2010) refiere, que es "una

\section{0
3
5
5
0
0
0}


técnica particular para aportar ayuda a los procedimientos de selección de las ideas primarias y secundarias". (p. 71). Por ello, se procedió a la utilización del subrayado, resúmenes, fichaje, como parte básica para la revisión y selección de los documentos que presentan el contenido teórico. Es decir, que mediante su aplicación de estas técnicas se pudo llegar a recoger informaciones en cuanto a la revisión bibliográfica de los diversos elementos encargados de orientar el proceso de investigación.

Tal como lo expresa, (Dávila, 2010, pág. 98)“las técnicas documentales proporcionan las herramientas esenciales y determinantes para responder a los objetivos formulados y llegar a resultados efectivos" ( $p$. 58). Es decir, para responder con eficiencia a las necesidades investigativas, se introdujeron como técnica de recolección el método inductivo, que hizo posible llevar a cabo una valoración de los hechos de forma particular para llegar a la explicación desde una visión general. Asimismo, se emplearon las técnicas de análisis de información para la realización de la investigación que fue ejecutada bajo la dinámica de aplicar diversos elementos encargados de determinar el camino a recorrer por el estudio,

Según (Dávila, 2010, pág. 99) las técnicas de procesamiento de datos en los estudios documentales "son las encargadas de ofrecer al investigador la visión o pasos que debe cumplir durante su ejercicio, cada una de ellas debe estar en correspondencia con el nivel a emplear" (p. 123). Esto indica, que para llevar a cabo el procesamiento de los datos obtenidos, es necesario establecer las técnicas que serán seleccionadas, destacándose en este caso, de manera particular: fichas de resumen, textual, registros descriptivos entre otros, los mismos se deben ajustar al nivel que ha sido seleccionado.

\section{Resultados}

\section{Atención integral de personas vulnera- bles en tiempo de pandemia}

En las últimas décadas, en muchos países de América Latina con gobiernos democráticos, fueron implementados nuevos modelos de Atención Primaria de Salud, APS, los cuales fueron implementados, "a través de una orientación territorial, comunitaria, familiar, intersectorial, participativa y visión hacia la conformación de sistemas universales de salud". (Naydú, 2020). Cabe señalar que este tipo de plan sanitario, conformado por estrategias y políticas comunitarias buscan acercarse al enfoque de APS integral concebido en Alma Ata, el cual, ante todo, pretende alejarse de un enfoque selectivo, "buscándose con el mismo, desarrollar un abordaje en salud que logre adaptarse a las necesidades territoriales, poblacionales e integrales de cada comunidad". (Naydú, 2020). Es por esta razón que, en respuesta de la amenaza y estragos causados por la presencia del Coronavirus se ha hecho necesario:

Llevar a cabo una mayor organización, planificación, consenso y participación ciudadana preventiva, aplicándose para tal fin, medidas integrales que involucran tanto acciones sanitarias, de comunicación e información, así como intervenciones económicas y sociales en apoyo de los sectores de la población vulnerables y de menores ingresos. (Naydú, 2020).

En virtud de esto, según (Tejerina, 2020), "se debe destacar que toda política pública con enfoque de derechos humanos para la prevención, atención y contención de la pandemia requiere un abordaje amplio y multidisciplinario". (p.12). El cual sea llevado a cabo, a partir del fortalecimiento de mecanismos de cooperación internacional entre estados, a fin de lograr eficacia de 
manera regional, global y sostenible en las políticas públicas y medidas de distinta naturaleza que se adopten.

Por todo esto, y en respuesta de lo acontecido a nivel mundial por el Coronavirus se hace necesario que las "distintas naciones del mundo entero logren establecer patrones en salud que garanticen los derechos de los grupos en situación de especial vulnerabilidad al momento de adoptar medidas de atención, tratamiento y contención de la pandemia de esta enfermedad" (Tejerina, 2020). Mitigando de esa forma, los impactos diferenciados que dichas medidas puedan generar, buscándose con ello, promover desde las más altas autoridades la eliminación de estigmas y estereotipos negativos que puedan surgir sobre ciertos grupos de personas a partir del contexto de pandemia.

Indudablemente que, es necesario "garantizar el control de una epidemia, además de garantizar la atención individual". (Domínguez, 2020), se necesita de un enfoque "integral, comunitario y territorial, de vigilancia y atención en salud", el cual permita que las acciones ejercidas por la Atención Primaria de la Salud APS incluyan, características de financiamiento y organización". (p. 5). Que vayan en correspondencia con las características generales de los sistemas de salud en cada país, los cuales incluyan elementos comunes: equipos multiprofesionales con enfoque familiar, comunitario y territorial.

Es en este sentido que la APS Integral e integrada toma un nuevo sentido y "reclama nuevos desarrollos que contribuyan a recobrar el equilibrio y la armonía entre sociedad y naturaleza desde los territorios". (Domínguez, 2020). Dicha especialidad en salud debe estar dirigida a la promoción, prevención, curación, rehabilitación y atención bío-psico-socio-cultural, la misma tiene un rol fundamental y debe ser el centro de los sistemas sanitarios de enfoque territorial colectivo, comunitario, centrados en el cui- dado integral de la persona, la familia y la comunidad.

Por esta razón, se ha podido observar que en la actual pandemia, "las prioridades sanitarias han dirigido sus estrategias de acción hacia actividades que busquen proteger a la población humana del contagio". (Wallace 2020). Utilizando métodos "que permitan identificar precozmente casos y contactos, con el fin de cortar la transmisión y atender integralmente tanto a enfermos como a personas aisladas, especialmente, los grupos de personas más vulnerables" (Domínguez, 2020), como lo son las personas con enfermedades crónicas, mujeres embarazadas, discapacitados, en fin todas aquellas que merezcan un atención médica primaria y especial.

Se puede decir entonces que, los sistemas mejor equipados para desarrollar eficazmente esas acciones "parecen ser los fundados sobre la Atención Primaria de Salud APS integral e integrada". (Wallace 2020), Los mismos, deben incluir "visión epidemiológica crítica, preocupada por las determinaciones sociales del complejo proceso transmisión, aislamiento, tratamiento y no solamente en contar casos y muertes". (Domínguez, 2020). Estos equipos deben incluir "prioritariamente a las personas mayores en los programas de respuesta a la pandemia, especialmente en el acceso a las pruebas de COVID-19, al tratamiento oportuno al acceso a medicamentos y a los cuidados paliativos necesarios. (Cueto 2020), garantizándose que brinden su consentimiento previo, pleno, libre e informado y teniendo en cuenta situaciones particulares como la pertenencia a pueblos indígenas o afrodescendientes.

Por todo lo señalado anteriormente, los equipos de Atención Primaria de Salud deben ante todo, adoptar las medidas necesarias a fin de prevenir los contagios de COVID-19 de la población mayor en general, personas con enfermedades crónicas, mujeres

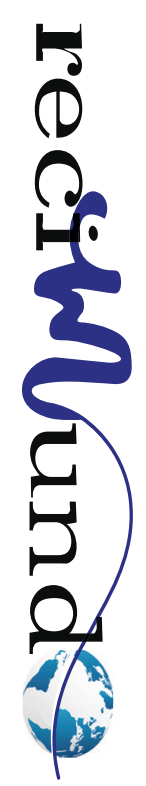


embarazadas, y en particular de aquellas quienes se encuentren en residencias de larga estancia, hospitales y centros de privación de libertad, "adoptando medidas de ayuda humanitaria que les permitan garantizarles la provisión de alimentos, agua y saneamiento y estableciendo espacios de acogida para personas en situación de pobreza extrema, calle o abandono o situación de discapacidad". (Domínguez, 2020)

\section{Conclusiones}

La llegada del Covid 19 en el mundo entero ha permitido observar que el enfoque sanitario dominante desde varios gobiernos y sistemas de salud para enfrentar la pandemia ha sido el biomédico. Este enfoque, por un lado, ha estado centrado en la enfermedad, con repuestas fundamentalmente hospital océntricas y con énfasis en el manejo médico individual; por el otro, en una vigilancia epidemiológica dedicada a contar casos, defunciones y hacer proyecciones descontextualizadas para predecir y mitigar las demandas de atención hospitalaria. De manera general, se pudo observar que en la mayoría de los países del mundo entero la respuesta inicial a la pandemia estuvo centrada en acciones curativas, biomédicas y hospitalocéntricas, con un vacío en la atención integral de enfoque promocional/ preventivo como primera medida de abordaje.

En efecto, tales acciones hicieron evidente que la preocupación fundamental hacia la propagación de esta pandemia estuvo basada en el tratamiento individual de la enfermedad, unidades de cuidados intensivos y el uso de respiradores, para lo cual se hizo especial énfasis en una estrategia epidemiológica que se dedica básicamente a dos tareas: por un lado, dictar, regular y controlar (con diferentes grados de rigidez y represión) cuarentenas sin tomar medidas suficientes para garantizar que la población con precarias condiciones de vida recibiera protección que les permitiera cumplir el distanciamiento social; por otro lado, contar casos y defunciones para observar el comportamiento de la curva de crecimiento de la epidemia en función de adecuar la respuesta médico asistencial.

No obstante, es de gran importancia entender que la prevención y control de una epidemiade grandes proporciones como lo es el Coronavirus, además de la asistencia médica individual, exige un abordaje centrado en las poblaciones, en especial aquellas que se encuentren en situaciones vulnerables, acción médica ésta que debe estar dirigida a proteger a estas personas del contagio, detectar precozmente sus síntomas, rastrear los contactos, aislar, tratar y rehabilitar a los afectados, con prioridad en los grupos sociales en desventaja. En definitiva, para poder enfrentar esta pandemia de manera efectiva, se requiere de un enfoque promocional, territorial, comunitario y de vigilancia integral en salud que puede ser desarrollado desde una estrategia de Atención Primaria de Salud (APS) Integral e Integrada, o lo que algunos vienen Ilamando Cuidado Integral de la Salud:

Por todo esto, se ha hecho fundamental que las distintas Naciones logren fijar medidas de prevención y mejora de la salud que sean ejecutadas por miembros y especialistas en Atención Primaria de la Salid APS, los cuales logren ejecutar parámetros de equidad, justicia, no discriminación, solidaridad y proporcionalidad, por ser éstos necesarios y decisivos en el establecimiento de un cuadro de toma de decisiones. Estos esquemas deben basarse fundamentalmente en la equidad y justicia como principios bioeticos.

Dichas medidas de atención y prevención deberán ser dirigidas a supervisar que los protocolos médicos, las decisiones sobre recursos médicos y tratamientos en relación 
al COVID-19 sean implementados sin discriminación en razón de la edad y prestando especial atención a las personas mayores con discapacidad o condiciones crónicas y enfermedades crónicas, pacientes con VIH o sida, que requieren medicación y atención regular como pacientes de diabetes, hipertensión, demencia senil, alzhéimer, entre otras.

Es decir, es importante que las políticas en salud ejecutadas por las autoridades sanitarias de cada país, busquen establecer el balance que debe existir entre la protección ante el COVID-19 y la necesidad particular que tienen las personas en situación vulnerable, facilitando medios alternativos médicos y de contacto familiar; todo esto, a partir de un enfoque interseccional en todas las respuestas de los Estados para contener la pandemia, teniendo en cuenta los distintos contextos y condiciones que potencializan la vulnerabilidad que caracterice cada persona y su situación; como lo es, la enfermedad que presente, condiciones médicas actuales del paciente, la precariedad económica, la edad, la condición de migrante o desplazada , la condición de discapacidad, la privación de libertad, el origen étnico-racial, la orientación sexual, identidad y/o expresión de género, entre otras.

Del mismo modo, estas medidas de prevención deben reforzar no sólo, el cuidado de las personas con enfermedades crónicas y en situación médica de cuidado, sino la protección de niños, niñas y adolescentes (NNA), incluyendo muy especialmente aquellos que no cuentan con cuidados familiares y que se encuentran en instituciones de cuidado, buscándose con ello, prevenir el contagio por el COVID-19, e implementar medidas que consideren sus particularidades como personas en etapa de desarrollo y que atiendan de manera más amplia posible su interés superior, garantizándose a su vez, los vínculos familiares y comunitarios.

\section{Bibliografía}

Arias, F. (2010). Paradigmas de la Investigación Científica. España: Editorial: Luces.

CIDH, C. I. (2020). Pandemis y derechos Humanos. Revista de Salud Integral de la Universidad Nacional de México, 6- 14.

Cueto, N. (2020). The origins of primary health care and selective primary health care.,. Review American Journal of PublicHealth.

Dávila, G. (2010). Técnicas de recolección y análisis de Datos. España: Editorial: Sintesis.

Domínguez, R. (2020). Abordaje del COVID en poblaciones vulnerables. Revista del Sistema de salud de Cuba. Salud Pública de México, Cuernavaca, 6- 13 .

Jacobo, H. (2020). Es la Atención Primartia de Salud Integral la respuesta a la Pandemis de Civis 19 en Latinoamérica. Revista Scielo. Río de Janeiro, 613.

Levín, C. y. (2020). Situación de los grupos vulnetables en medio de la Pandemia. Revista EI CISNE. Centro Educativo Terapéutico de las Américas, 8 $-29$.

Naydú, R. (2020). Evaluación de un modelo de atención primaria en salud en Santander, Colombia. Revista Facultad Nacional de Salud Pública, Bogotá, v. 34, n. 1, p. 88-95, 34- 91.

Tejerina, A. y. (2020). de la Atención Reformas de la Atención Primaria Integral de Salud. Revista de Medicina Social, New York, 12- 23.

Wallace, C. (2020). Big farms make big flu: dispatches on infectious diseas. Review American Journal of Public Health. 


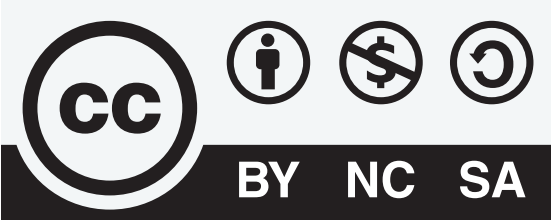

CREATIVE COMMONS RECONOCIMIENTO-NOCOMERCIAL-COMPARTIRIGUAL 4.0.

\section{CITAR ESTE ARTICULO:}

Baquerizo Suárez, P. J., Zamora Baquerizo, D. A., \& Moreira Cedeño, L. D. (2021). Búsqueda activa de personas vulnerables para garantizar una atención integral en tiempos de pandemia. RECIMUNDO, 5(1), 90-98. https://doi. org/10.26820/recimundo/5.(1).enero.2021.90-98 